\title{
Nonstationary two-level resonance fluorescence $\uparrow$
}

\author{
B Renaud, R M Whitley and C R Stroud Jr \\ The Institute of Optics, University of Rochester, Rochester, NY 14627, USA
}

Received 19 July 1976, in final form 14 September 1976

\begin{abstract}
Analytic expressions are obtained for the spectrum of the light scattered when a collimated atomic beam is illuminated at right angles to its path by a $\mathrm{CW}$ monochromatic laser beam tuned to resonance with a two-level transition. The spectra, as would be determined by a Fabry-Perot interferometer, vary depending on the portion of the interaction region from which the scattered light emanates. The spectrum of the light from a finite sub-region of the interaction volume is described as a function of the location and length of the sub-region, and of the intensity and frequency of the laser. Several interesting features related to the turn-on of the interaction as well as to the finite observation interval are found.
\end{abstract}

\section{Introduction}

Consider an atomic beam illuminated at right angles to its path by a $\mathrm{CW}$ monochromatic laser beam tuned to resonance with a two-level transition. The atoms will strongly scatter the incident light. The spectrum of that spontaneously emitted radiation exhibits distinctive features due to the AC Stark effect. This spectrum, as a function of the laser's intensity and its detuning from the two-level resonance, has been the subject of a number of theoretical papers (Mollow 1969, 1975, Herrmann et al 1973, Agarwal 1974, Hassan and Bullough 1975, Smithers and Freedhoff 1974, Carmichael and Walls 1975, 1976, Cohen-Tannoudji 1975, Swain 1975, Kimble and Mandel 1976). This spectrum has also been measured in four different laboratories in the past three years (Schuda et al 1974, Walther 1975, Wu et al 1975, Gibbs and Venkatesan 1976). In this paper, we derive in detail the expression for the spectrum of the scattered light as measured by means of a Fabry-Perot interferometer, using the results of a previous quantum electrodynamic calculation for the relevant correlation function (Renaud et al 1976, to be referred to as RWS I). This expression is found to be a well behaved positive definite function of the scattered frequency, as one would expect for such a quantity.

Our analysis describes measurements of the spectrum of the light emitted by the ensemble of atoms comprising any given part of the atomic beam (henceforth referred to as the observation region). Our results apply even in the transient regime. Of particular interest is the region where the atoms in an arbitrary initial state enter the laser field, and thereby experience the sudden turn-on of this interaction. We show that these spectra exhibit features that are not present in the stationary limit,

† This work was supported in part by the ONR through Contract N0014-68-A-0091. 
which corresponds to an observation region of infinite length. These features, which we will characterize as 'transient', can be attributed either to the finite length of the observation region or to the recent start of the interaction. Specifically, a finite observation region (in which each atom spends only a limited amount of time) leads to a general broadening of the spectrum and, in most instances, to oscillations near the field frequency. The transient effects observed near the beginning of the interaction region are very sensitive to the choice of the initial state of the atom. The expression we derive for the spectrum allows one to prescribe any initial condition; however, for the most part our attention is directed to atoms which are in the ground state as they enter the laser field, since this is the situation most easily realized experimentally. In such cases quite asymmetrical lineshapes can be obtained for off-resonance driving fields, in contrast to the symmetrical ones obtained from observations of only those atoms which have been in the interaction region for many lifetimes. In the limit where the atom never becomes appreciably excited these asymmetrical spectra are found to be exactly those predicted by the Lorentz model.

The above-mentioned transient features have not generally been found in other treatments because assumptions were made which are equivalent to assuming that each atom is observed interacting with the field for an infinite amount of time. However, some results for the transient regime have been published. Herrmann et al (1973) solved for the spectrum under the condition that each atom interacts with the field for only a finite amount of time. Carmichael and Walls (1976) have given approximate (strong-field) solutions allowing for a finite observation time, both for an atom in the steady state and for an excited atom observed entering the interaction region. The situation we treat is slightly more general in that it allows for a finite interval between the start of the interaction and the observation. Moreover, we allow the laser field to be detuned from exact resonance. In contrast, both of the above treatments are restricted to exactly resonant fields, where the usual initial conditions of a pure excited state or a pure ground state result in a symmetric spectrum.

It should be noted that, even though the interaction of a given atom with the field is not stationary, the spectrum of the light scattered from any part of the interaction region is time independent. This is a consequence of the assumption that the atoms are passing through the incident radiation field at a constant rate.

\section{Experimental arrangement}

In figure 1 the geometry of the idealized experiment is shown. The atomic beam, which is well collimated, is along the $y$ axis, while the laser beam is along the $x$ axis. The spectrum of the scattered light will be measured in a small solid angle about the $z$ direction. For simplicity the laser beam is taken to have a uniform intensity over a rectangular cross section. In a real experiment this might be approximated by spreading the laser beam and then using an aperture near the atomic beam to truncate the Gaussian, leaving a reasonably uniform intensity distribution. As is indicated in figure $1(b)$, we assume that a slit is placed above the atomic beam so as to allow observation of only a portion of the interaction region at a time. The beginning of the interaction region is at $y=0$, while the observation region is bounded by $y_{1}$ and $y_{2}$. It is also convenient to introduce a time parameter $T$ for a particular atom. If the atom is moving with a velocity $v$ along the beam then it will require a time $T_{1}$ to travel from the beginning of the interaction region to 


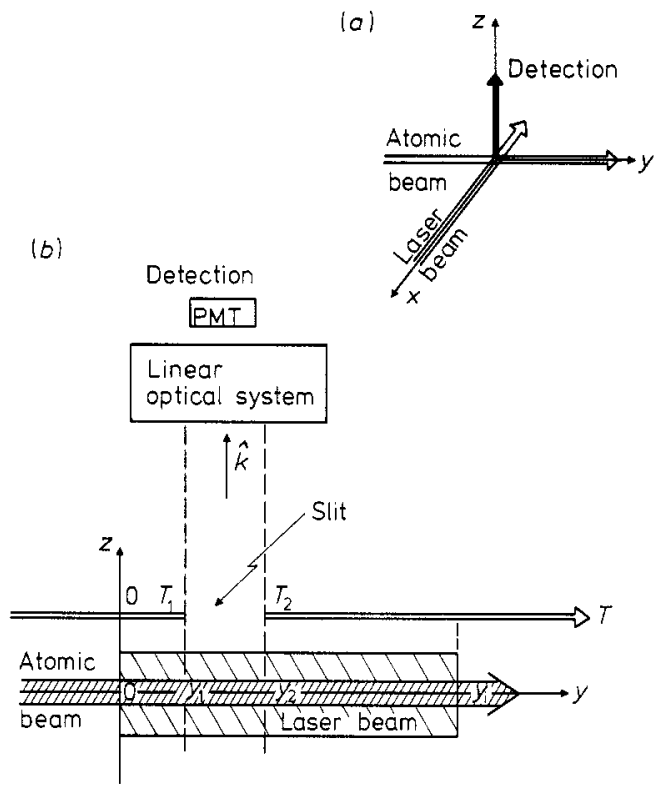

Figure 1. (a) The geometry of the idealized experiment. (b) The atomic beam and the $\mathrm{cW}$ laser interact in a volume which has a rectangular cross section in the $y z$ plane. The part of the interaction region visible to the detector is limited to the observation region $\left[y_{1}, y_{2}\right]$ by the slit.

the beginning of the observation region. It will leave the observation region at $T_{2}$ and, in general, will satisfy the relation $y=v T$. The length of this interval will be denoted by $\tau=T_{2}-T_{1}$.

The scattered light passes through a linear optical system and is detected by a photomultiplier. Though our analysis applies for any linear system, we will specialize to an ideal Fabry-Perot.

Consider one atom in the observation region $\left[y_{1}, y_{2}\right]$. The expression for the intensity (power per unit solid angle) in the far field zone can be written in terms of the positive and negative frequency parts of the field as (see Rehler and Eberly 1971)

$$
I_{v}\left(\hat{\boldsymbol{k}}, t-t_{0}+r / c\right)=\frac{r^{2} c}{2 \pi}\left\langle\boldsymbol{E}^{(-)}\left(t-t_{0}+r / \mathcal{c}\right) . \boldsymbol{E}^{(+)}\left(t-t_{0}+r / c\right)\right\rangle
$$

Here the subscript $v$ refers to the velocity $v$ of the atom, $t_{0}$ to the time when it enters the laser radiation, and $r$ to the separation between the detector and the interaction region. The direction is denoted by the unit vector $\hat{k}$. The positive and negative frequency parts of the field satisfy

$$
\boldsymbol{E}^{( \pm)}\left(t-t_{0}+r / c\right) \equiv \begin{cases}\boldsymbol{E}^{( \pm)}(T+r / c) \neq 0 & \text { for } T \in\left[T_{1}, T_{2}\right] \\ 0 & \text { otherwise. }\end{cases}
$$

That is, the atom contributes to the detected field only when it is situated within the observation region. Then the intensity emanating from the interval $\mathrm{d} y$ about the coordinate $y$ in the observation region is just the average of $I_{v}(\hat{\boldsymbol{k}}, T+r / c)=I_{v}(\hat{\boldsymbol{k}}$, $y / v+r / c)$ over the velocity distribution $P(v)$ for the atoms in the beam, multiplied 
by the constant density of atoms $N_{\mathrm{a}}$, by the cross section $\mathscr{A}$ of the atomic beam and the differential length $\mathrm{d} y$. We neglect all cooperative effects between the atoms.

As a second step, this intensity can be integrated over $y$ from $y_{1}$ to $y_{2}$ to yield the total intensity emitted from all $N$ atoms within the observation region $\left(N=N_{\mathrm{a}} \mathscr{A}\left(y_{2}-y_{1}\right)\right)$, i.e.

$$
I\left(\hat{k}, y_{1}, y_{2}\right)=N \int \mathrm{d} v P(v) \frac{1}{T_{2}-T_{1}} \int_{y_{1} / v}^{y_{2} / v} I_{v}(\hat{k}, T+r / c) \mathrm{d} T .
$$

Due to the average over the ensemble of atoms within the observation region, this total intensity is time independent, although the contribution of the individual atoms to the intensity, as in (1), is not.

Relation ( $3 a$ ) takes into account the velocity distribution in the atomic beam. If we assume that all atoms travel at the same velocity $\bar{v}$, then $(3 a)$ reduces to

$$
I_{\mathrm{D}}\left(\hat{\boldsymbol{k}}, T_{1}, T_{2}\right)=I\left(\hat{\boldsymbol{k}}, \bar{v} T_{1}, \bar{v} T_{2}\right)=\frac{N r^{2} c}{2 \pi \tau} \int_{T_{1}}^{T_{2}} \mathrm{~d} T\left\langle\boldsymbol{E}^{(-)}(T+r / c) \cdot \boldsymbol{E}^{(+)}(T+r / c)\right\rangle
$$

where we used $\tau=T_{2}-T_{1}$. This is the correct expression to use if the atomic beam is velocity selected (Schuda and Stroud 1973).

It is convenient to define the truncated Fourier transform

$$
\widetilde{E}^{( \pm)}(\omega)=\int_{T_{1}}^{T_{2}} \mathrm{~d} T E^{( \pm)}(T+r / c) \mathrm{e}^{ \pm \mathrm{i} \omega T}
$$

where $\omega$ is a positive (real) frequency. The superscripts ' \pm ' are used here to show how both $E^{(+)}(t)$ and $E^{(-)}(t)$ are transformed. The inverse of $(4 a)$ is

$$
E^{( \pm)}(T+r / \mathcal{c})= \begin{cases}\frac{1}{2 \pi} \int_{0}^{\infty} \mathrm{d} \omega \tilde{E}^{( \pm)}(\omega) \mathrm{e}^{\mp i \omega T} & T \in\left[T_{1}, T_{2}\right] \\ 0 & \text { otherwise }\end{cases}
$$

The last two expressions, when substituted into $(3 b)$, yield

$$
I_{\mathrm{D}}\left(\hat{\boldsymbol{k}}, T_{1}, T_{2}\right)=\frac{N r^{2} c}{(2 \pi)^{2} \tau} \int_{0}^{\infty}\left\langle\tilde{\boldsymbol{E}}^{(-)}(\omega) \cdot \tilde{\boldsymbol{E}}^{(+)}(\omega)\right\rangle \mathrm{d} \omega .
$$

Here $\tilde{\boldsymbol{E}}^{(+)}(\omega)\left(\tilde{\boldsymbol{E}}^{(-)}(\omega)\right)$ is interpreted as the $\omega$ component of the positive (negative) frequency part of the field transmitted by the linear optical system.

This time-independent linear system (see figure 1) transforms the frequency components of the incident field according to the product

$$
\tilde{E}^{(+)}(\omega)=\mathbf{Y}(\omega) \cdot \tilde{E}_{\mathrm{i}}^{(+)}(\omega)
$$

In particular, the transmission function $\mathbf{\Upsilon}(\omega)$ for a non-absorbing plane Fabry-Perot interferometer (Born and Wolf 1970) is the $3 \times 3$ matrix

$$
\mathbf{r}(\omega)=\mathbf{I}\left(\frac{1-R}{1-R \mathrm{e}^{\mathrm{i} \omega \tau^{\mathrm{FP}}}}\right)
$$

where $I$ is the $3 \times 3$ unit matrix and $R$ is the reflectivity of the Fabry-Perot plates. Relation (7a) says that each vectorial component (as well as each frequency component) is transformed independently. The phase difference in the exponential is the 
product of the frequency $\omega$ and the time delay $\tau^{\mathrm{FP}}$ between two successive transmitted waves

$$
\tau^{\mathrm{FP}}=2(1 / \mathrm{c}) n h \cos \theta
$$

Here, $n$ is the refractive index of the medium between the plates, $h$ is their separation and $\theta$ is the angle of incidence.

The expression for $E_{i}^{(+)}(T+r / c)$ in the far field zone of the atom is written as the sum of the free field and the dipole field (Rehler and Eberly 1971, Mollow 1969)

$$
\boldsymbol{E}_{\mathrm{i}}^{(+)}(T+r / c) \simeq \boldsymbol{E}_{\mathrm{i} 0}^{(+)}(T+r / c)+\frac{\Omega^{2}}{r c^{2}} \sum_{\lambda} \hat{\boldsymbol{\epsilon}}_{\hat{\boldsymbol{k}} \lambda}\left(\hat{\boldsymbol{\epsilon}}_{\hat{\boldsymbol{k}} \lambda}^{*} \cdot \boldsymbol{\mu}^{*}\right) \sigma_{-}(T)
$$

where $\Omega$ is the atomic transition frequency and $\mu$ the dipole-moment matrix element. The polarization unit vector is denoted by $\hat{\boldsymbol{\epsilon}}_{\hat{k} \lambda}, \hat{\lambda}=1,2$. Also $\sigma_{-}(T)$ is the Heisenberg atomic lowering operator. The atomic raising operator appears in the corresponding expression for $\boldsymbol{E}_{\mathrm{i}}^{(-)}(T+r / c)$.

This far field $E_{\mathrm{i}}^{(+)}(T+r / c)$ can be Fourier transformed according to equations (4). By doing so, and using relations (5) to (8), the total transmitted intensity becomes a triple integral expression whose integrand contains an autocorrelation function:

$$
\begin{aligned}
I_{\mathrm{D}}\left(\hat{\boldsymbol{k}}, T_{1}, T_{2}\right) & \simeq \frac{N \Omega^{4}}{(2 \pi)^{3} c^{3} \tau} \sum_{\hat{\lambda}}\left(\hat{\boldsymbol{\epsilon}}_{\hat{\boldsymbol{k}} \lambda} \cdot \mu\right)\left(\hat{\boldsymbol{\epsilon}}_{\hat{\mathbf{k}} \lambda}^{*} \cdot \mu^{*}\right) \int_{0}^{\infty} \mathrm{d} \omega\left[1+\frac{4 R}{(1-R)^{2}} \sin ^{2}\left(\frac{\omega \tau^{\mathrm{FP}}}{2}\right)\right]^{-1} \\
& \times \int_{T_{1}}^{T_{2}} \mathrm{~d} t^{\prime \prime} \int_{T_{1}}^{T_{2}} \mathrm{~d} t^{\prime}\left\langle\sigma_{+}\left(t^{\prime}\right) \sigma_{-}\left(t^{\prime \prime}\right)\right\rangle \mathrm{e}^{-\mathrm{i} \omega\left(t^{\prime}-t^{\prime \prime}\right)}
\end{aligned}
$$

The two time integrals in the double truncated Fourier transform of the atomic correlation function $\left\langle\sigma_{+}\left(t^{\prime}\right) \sigma_{-}\left(t^{\prime \prime}\right)\right\rangle$ are taken with respect to the same conjugate variable $\omega$. The remaining integral over $\omega$ involves an Airy function whose form can be derived directly from $(7 a)$. This function describes the filtering action of the FabryPerot interferometer. Its transmission peaks (of area $A$ and unit height) occur at the frequencies

$$
\omega_{f}=\frac{2 \pi f}{\tau^{\mathrm{FP}}}=\frac{f \pi c}{n h \cos \theta}
$$

where $f$ is a large positive integer.

We assume we have an ideal Fabry-Perot. The reflectivity $R$ of its plates is nearly one, and its free spectral range is much larger than the width of the frequency spectrum of the atomic correlation function. In this case equation (9), the total intensity measured by the detector, becomes

$$
\begin{aligned}
& I_{\mathrm{D}}\left(\hat{\boldsymbol{k}}, T_{1}, T_{2}, \omega_{f}\right)=\frac{N \Omega^{4} A}{\tau(2 \pi)^{2} c^{3}} \sum_{\lambda}\left(\hat{\boldsymbol{\epsilon}}_{\hat{\boldsymbol{k}} \lambda} \cdot \boldsymbol{\mu}\right)\left(\hat{\boldsymbol{\epsilon}}_{\hat{\boldsymbol{k}} i}^{*}, \boldsymbol{\mu}^{*}\right) \int_{T_{1}}^{T_{2}} \mathrm{~d} t^{\prime \prime} \int_{T_{1}}^{T_{2}} \mathrm{~d} t^{\prime}\left\langle\sigma_{+}\left(t^{\prime}\right) \sigma_{-}\left(t^{\prime \prime}\right)\right\rangle \\
& \times \exp \left[-\mathrm{i} \omega_{f}\left(t^{\prime}-t^{\prime \prime}\right)\right] .
\end{aligned}
$$

By slightly changing $h$, the separation of the plates, $\omega_{f}$ would change by a value smaller than the free spectral range, scanning over the whole atomic spectrum. 
Relation (11) is the final expression for the total intensity at frequency $\omega_{f}$ coming from the observation region and measured by the detector in the far field zone.

\section{Analysis}

In $\$ 2$ we have found that the intensity at the detector depends upon the autocorrelation of the atomic lowering operator. We wish to express this correlation function explicitly in terms of the atomic and field parameters. This is readily done by using the results of our previous paper (RWS I) in which it was shown that this correlation function can be written in the form

$\left\langle\sigma_{+}\left(t^{\prime}\right) \sigma_{-}\left(t^{\prime \prime}\right)\right\rangle=\sum_{l, m=1}^{4} C_{l m} \exp \left[\left(s_{l}-\mathrm{i} \omega_{0}\right) t^{\prime}\right] \exp \left[\left(s_{m}-s_{l}+\mathrm{i} \omega_{0}\right) t^{\prime \prime}\right]$.

Here the times are ordered by the convention

$$
t^{\prime \prime}-t^{\prime}>0
$$

The coefficients $C_{l m}$ have the explicit form

$$
\begin{aligned}
& C_{l m}=\frac{1}{D\left(s_{l}\right) D\left(s_{m}\right)} \llbracket\left[s _ { l } [ ( s _ { l } + 2 \beta ) ( s _ { l } + \beta + \mathrm { i } \alpha ) + \frac { 1 } { 2 } | \epsilon | ^ { 2 } ] \left\{\langle \sigma _ { 2 2 } ( 0 ) \rangle \left[\left(\alpha^{2}+\left(s_{m}+\beta\right)^{2}\right) s_{m}\right.\right.\right. \\
&\left.+\frac{1}{2}|\epsilon|^{2}\left(s_{m}+\beta\right)\right]-\left\langle\sigma_{-}(0)\right\rangle \frac{1}{2} \epsilon^{*}\left(s_{m}+\beta+\mathrm{i} \alpha\right) s_{m} \\
&\left.-\left\langle\sigma_{+}(0)\right\rangle \frac{1}{2} \epsilon\left(s_{m}+\beta-\mathrm{i} \alpha\right) s_{m}+\left\langle\sigma_{11}(0)\right\rangle \frac{1}{2}|\epsilon|^{2}\left(s_{m}+\beta\right)\right\} \\
&+\frac{1}{2} \epsilon\left(s_{l}+2 \beta\right)\left(s_{l}+\beta+\mathrm{i} \alpha\right)\left\{-\left\langle\sigma_{22}(0)\right\rangle \frac{1}{2} \epsilon^{*}\left(s_{m}-2 \beta\right)\left(s_{m}+\beta-\mathrm{i} \alpha\right)\right. \\
&+\left\langle\sigma_{-}(0)\right\rangle \frac{1}{2} \epsilon^{* 2} s_{m}-\left\langle\sigma_{+}(0)\right\rangle s_{m}\left[\left(s_{m}+2 \beta\right)\left(s_{m}+\beta-\mathrm{i} \alpha\right)+\frac{1}{2}|\epsilon|^{2}\right] \\
&\left.\left.+\left\langle\sigma_{11}(0)\right\rangle \frac{1}{2} \epsilon^{*}\left(s_{m}+2 \beta\right)\left(s_{m}+\beta-\mathrm{i} \alpha\right)\right\}\right]
\end{aligned}
$$

where in the denominator there appears the polynomial

$$
D(s)=4 s^{3}+12 \beta s^{2}+2 s\left(5 \beta^{2}+\alpha^{2}+|\epsilon|^{2}\right)+\beta\left(2 \beta^{2}+2 \alpha^{2}+|\epsilon|^{2}\right) .
$$

Also $\epsilon, \alpha$ and $\beta$ are respectively the on-resonance Rabi frequency, the detuning of the incident field with respect to the atomic frequency, and half the Einstein $A$ coefficient. The expectation values $\left\langle\sigma_{22}(0)\right\rangle$ and $\left\langle\sigma_{11}(0)\right\rangle$ are the initial populations of the excited and lower states respectively. The variables $s_{l}(l=1, \ldots, 4)$ are the four eigenvalues of the problem (see RWS I). Since one of these roots is trivially zero, let

$$
s_{1}=0
$$

The other three have negative real parts satisfying

$$
-2 \beta \leqslant \operatorname{Re}\left(s_{l}\right) \leqslant-\beta \quad l=2,3,4 .
$$


After making use of equations (12), (13) and (16), the total intensity measured by the detector, as given by (11), becomes:

$$
\begin{aligned}
& I_{\mathrm{D}}\left(\hat{\boldsymbol{k}}, T_{1}, T_{1}+\tau, \omega_{0}+\Delta\right) \\
&=\frac{N \Omega^{4} A}{(2 \pi)^{2} c^{3}} \sum_{\hat{\lambda}}\left(\hat{\boldsymbol{\epsilon}}_{\hat{\boldsymbol{k}} \lambda} \cdot \boldsymbol{\mu}\right)\left(\hat{\boldsymbol{\epsilon}}_{\hat{\boldsymbol{k}} \lambda}^{*}, \mu^{*}\right) \\
& \times\left[\sum_{l=1}^{4} \frac{C_{l 1}}{\left(s_{l}+\mathrm{i} \Delta\right)}\left(\frac{\exp \left[\left(s_{l}+\mathrm{i} \Delta\right) \tau\right]-1}{\left(s_{l}+\mathrm{i} \Delta\right) \tau}-1\right)+\mathrm{CC}\right. \\
&\left.+\sum_{l=1}^{4} \sum_{m=2}^{4} \frac{C_{l m} \exp \left(s_{m} T_{1}\right)}{\left(s_{l}-s_{m}+\mathrm{i} \Delta\right)}\left(\frac{\exp \left[\left(s_{l}+\mathrm{i} \Delta\right) \tau\right]-1}{\left(s_{l}+\mathrm{i} \Delta\right) \tau}-\frac{\exp \left(s_{m} \tau\right)-1}{s_{m} \tau}\right)+\mathrm{CC}\right]
\end{aligned}
$$

where the frequency variable

$$
\Delta=\omega_{f}-\omega_{0}
$$

is just the frequency of the scattered light transmitted through the Fabry-Perot interferometer, expressed with respect to that of the incident field.

Relation (18) is a well behaved positive function of $T_{1}, \tau$ and $\Delta$ as long as $\tau$ is finite. When the length $\tau$ of the observation region is allowed to go to infinity, then the double sum in equation (18) vanishes and $I_{\mathrm{D}}$ becomes independent of $T_{1}$. In this limit the spectrum $I_{\mathrm{D}}$ reduces to

$$
\begin{aligned}
I_{\mathrm{D}}\left(\hat{\boldsymbol{k}}, T_{1}, T_{1}\right. & \left.+\infty, \omega_{0}+\Delta\right) \\
& =\frac{N \Omega^{4} A}{(2 \pi)^{2} c^{3}} \sum_{\dot{\lambda}}\left(\hat{\boldsymbol{\epsilon}}_{\hat{\boldsymbol{k}} \lambda} \cdot \boldsymbol{\mu}\right)\left(\hat{\boldsymbol{\epsilon}}_{\hat{\boldsymbol{k}} \lambda}^{*} \cdot \boldsymbol{\mu}^{*}\right)\left(\sum_{l=2}^{4} \frac{-C_{l 1}}{\left(S_{l}+\mathrm{i} \Delta\right)}+\mathrm{CC}+2 \pi C_{11} \delta(\Delta)\right) .
\end{aligned}
$$

Because the $C_{l 1}$ are independent of the initial atomic conditions (cf equations (14) and (16)), this spectrum does not depend upon the state of the atoms as they enter the interaction region. This is as expected, since any finite length of the atomic beam near the point where the atoms enter the laser field now represents an arbitrarily small fraction of the entire observation region. Expression (20) is exactly the emission spectrum in the stationary limit found previously (Mollow 1969).

\section{Discussion of spectra}

The emission spectrum of a driven two-level atom is completely described by equation (18). This expression is valid for observations of atoms for which the correlation function $\left\langle\sigma_{+}\left(t^{\prime}\right) \sigma_{-}\left(t^{\prime \prime}\right)\right\rangle$ has become stationary and depends only on the time difference $t^{\prime}-t^{\prime \prime}$. However it is superior to expression (20) and similar results of previous treatments that assumed stationarity for $\left\langle\sigma_{+}\left(t^{\prime}\right) \sigma_{-}\left(t^{\prime \prime}\right)\right\rangle$ because it correctly incorporates the effect that an observation region of finite length has on the spectrum. More importantly, relation (18) is valid for observations of atoms which have just entered the laser field, and, therefore, cannot be described by a stationary correlation function. A number of interesting features, related to this sudden turn-on of the interaction and to the finite observation region are present in this spectrum (18). These are 
most easily understood with the aid of graphs. The intensity at the detector as expressed in equation (18) has been plotted in figures $2-6$ for different observation regions, and various values of the parameters characterizing the incident field.

As the laser is tuned away from resonance, the $C_{11}$ term in the expression for the intensity at the detector, equation (18), becomes increasingly important. We shall refer to this term as the elastic component of the spectrum, because in the stationary limit (i.e. when $\tau$ goes to infinity) it becomes a delta function at the frequency of the incident field. The behaviour of this component as a function of detuning is apparent from the sequence of emission spectra shown in figures $2(a)$ and $(b)$. For each graph, $\epsilon=10 \beta$ and the detuning $\alpha$ varies from 0 to $12 \beta$. Only the length of the observation region differs in the two figures.

Figure 2(a) shows the spectra in the stationary limit as given by relation (20). In order to make the elastic component visible in this graph, the delta function has been convolved with a Gaussian with a $1 / \mathrm{e}$ half-width $\Gamma$ of $\frac{1}{5} \beta$, a tenth of the
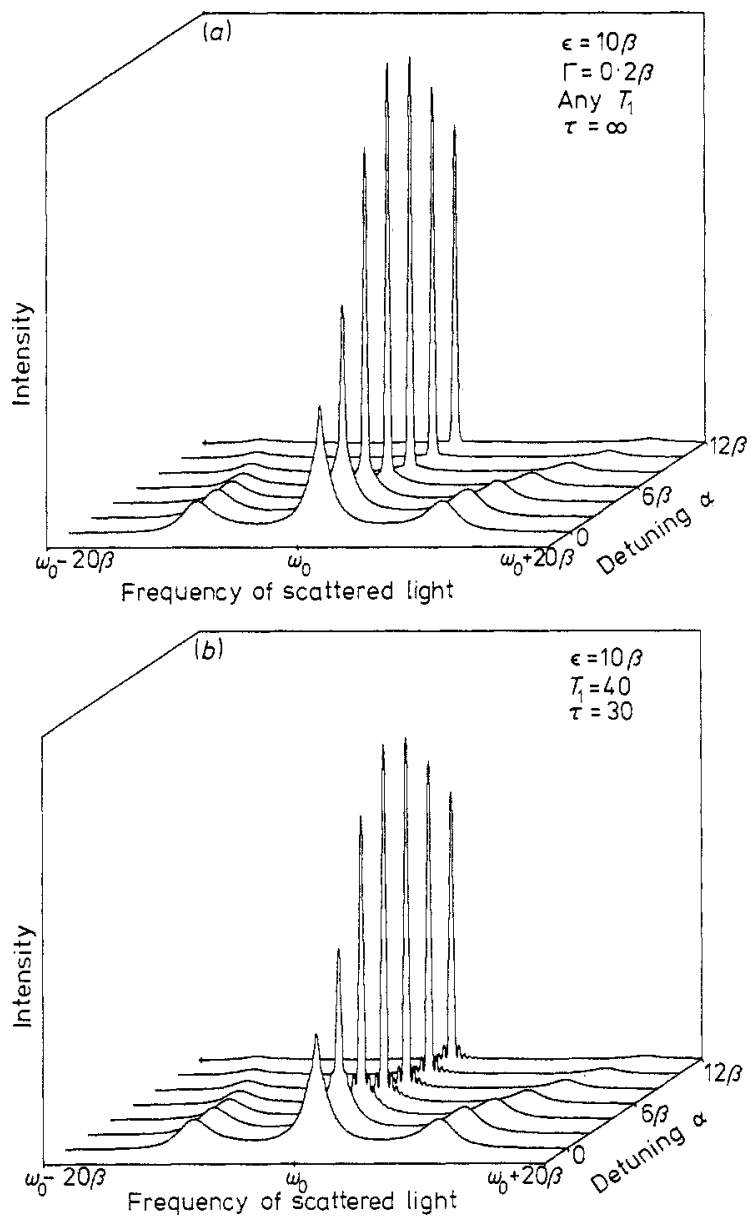

Figure 2. (a) Emission spectra in the stationary limit for various values of the detuning . The observation region is of infinite length. The delta function of the elastic component is convolved with a Gaussian with a $1 / \mathrm{e}$ half-width $\Gamma$ of $\frac{1}{5} \beta$. (b) Emission spectra for various values of the detuning $x$ in the case where the observation region corresponds to $\tau=30$ lifetimes. 
natural linewidth. The intensity in the elastic component is easily shown to be proportional to $\left.\left\langle\sigma_{-}(t=\infty)\right\rangle\right|^{2}$, which is given by

$$
\left|\left\langle\sigma_{-}(t=\infty)\right\rangle\right|^{2}=\frac{|\epsilon|^{2}\left(\beta^{2}+\alpha^{2}\right)}{\left(|\epsilon|^{2}+2 \beta^{2}+2 \alpha^{2}\right)^{2}} .
$$

This implies that the elastic component will be maximum at $\alpha=0$ for $|\epsilon| \leqslant \sqrt{2} \beta$, and has maxima at $\alpha= \pm\left(\frac{1}{2}|\epsilon|^{2}-\beta^{2}\right)^{1 / 2}$ for $|\epsilon| \geqslant \sqrt{2} \beta$. For the parameters of figure $2(a)$, this maximum occurs at $\alpha=7 \beta$.

It is realistic to assume that each atom in the beam is observed for only a finite amount of time, so in figure $2(b)$ the corresponding spectra from an observation region 30 lifetimes long are shown. The parameter $T_{1}$ has been chosen to be very large, which means that the atoms have been in the interaction region for many lifetimes before they enter the observation region. This ensures that no effects remain which are due to the turn-on of the field. One notices that the sinc oscillations near the elastic peak are small but still visible for non-resonant fields. More importantly, the width of the elastic component is still significant, since the half-width at half maximum is about $1 \cdot 77 \pi / \tau$, or $0.186 \beta$ in the particular case of figure $2(b)$.

The spectrum for the situation of a strong resonant field with no detuning has been widely discussed in the stationary limit $(\tau \rightarrow \infty)$. In figures $3(a)$ and $(b)$, we show the evolution of this spectrum as a function of the time the atoms spend in the field (see also Herrmann et al 1973, Carmichael and Walls 1976). The slit is adjusted so that the light is collected from atoms which have been in the field for times ranging from 0 up to $\tau$. This parameter $\tau$ is plotted on the axis going 'into' the page, in units of the natural atomic lifetime, $1 /(2 \beta)$. The atoms are assumed to be in the ground state when they enter the interaction region $(T=0)$. From an initially broad featureless spectrum, the central peak and the sidebands emerge rather quickly. When $\epsilon=4 \beta$, as in figure 3(a), they take shape after 10 lifetimes, whereas for a stronger field as in figure $3(b)$ where $\epsilon=10 \beta$, the sidebands are already visible at $\tau=2$. As the observation region is lengthened, all three components get taller and narrower. Then for about $\tau=15$ and $\tau=30$ respectively, the elastic component has narrowed enough to be distinguishable from the broader peak of the central inelastic component. Here, the delta function which is present in the stationary limit of both figures $3(a)$ and $(b)$ has been convolved with a Gaussian with a 1/e half-width $\tau$ of $\frac{1}{10} \beta$. Note that the delta function is a smaller fraction of the total spectrum for the stronger field.

In the limit of a very strong, exactly resonant field $(|\epsilon| \gg \beta)$, it is possible to simplify the expression (18) for the spectrum and illustrate explicitly the manner in which the sidebands and the inelastic central peak narrow with increasing $\tau$. In this limit, the eigenvalues become

$$
s_{1}=0, \quad s_{2}=-\beta, \quad s_{3}=s_{4}^{*}=-\frac{3}{2} \beta+i|\epsilon| .
$$

For an atom initially in the ground state, the coefficients simplify to

$\frac{1}{2} C_{21}=C_{31}=C_{41}=-C_{23}=-C_{24}=-C_{33}=-C_{44}=\frac{1}{8}+\mathscr{C}(\beta / \epsilon \mid)$

with all of the other coefficients of higher order. For $\tau$ greater than a few lifetimes, 

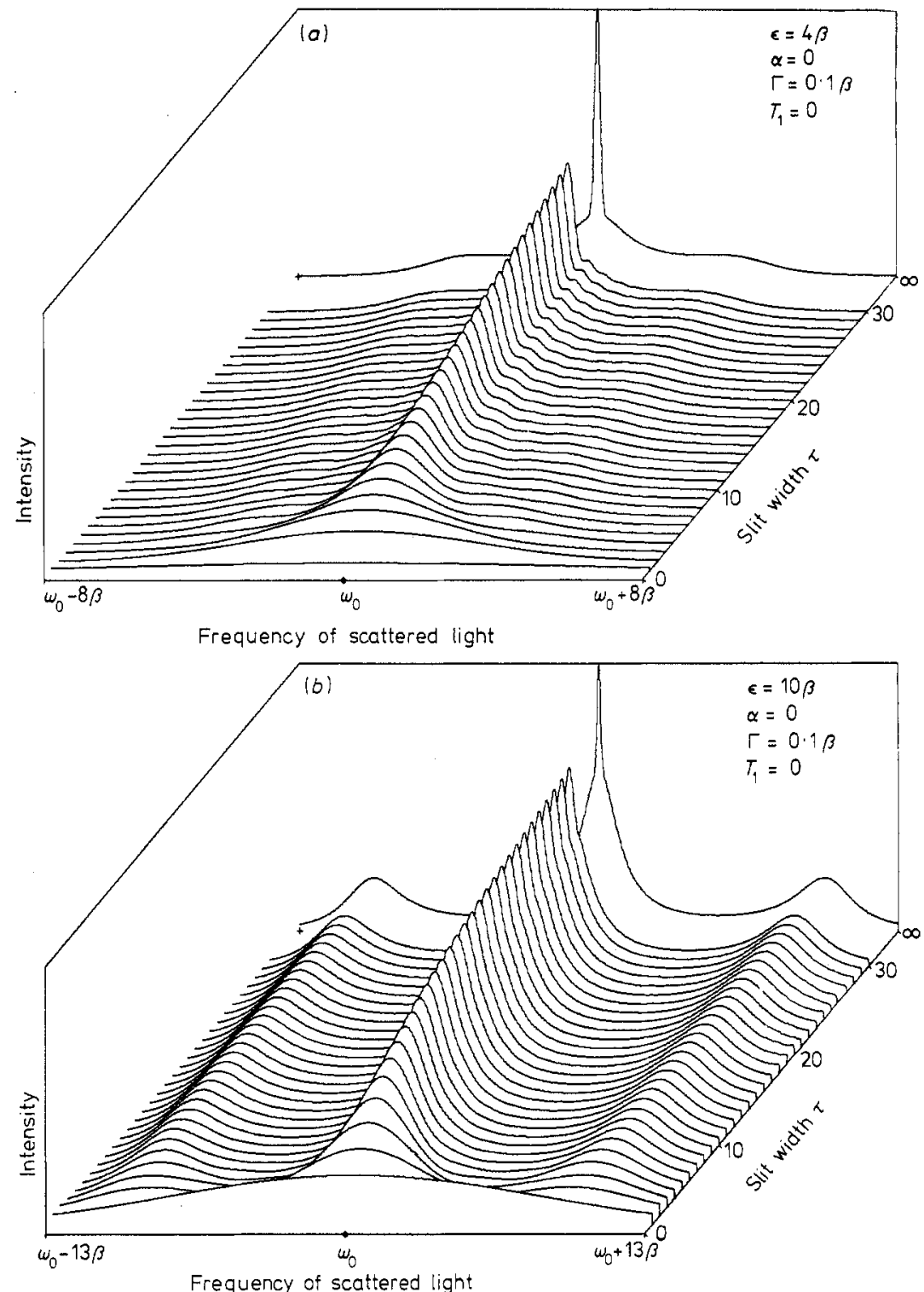

Figure 3. Emission spectra for various observation slit widths $\tau$ in the case of $(a)$ a moderately strong on-resonance field and $(b)$ a strong on-resonance field.

one finds that

$$
\begin{aligned}
I_{\mathrm{D}}\left(\hat{k}, T_{1}, T_{1}\right. & \left.+\tau, \omega_{0}+\Delta\right) \\
\propto & \propto \frac{1}{8 \tau}\left[\frac{4}{\beta^{2}+\Delta^{2}}\left(\beta \tau-\frac{\beta^{2}-\Delta^{2}}{\beta^{2}+\Delta^{2}}\right)\right. \\
& +\frac{2}{9 \beta^{2} / 4+(\Delta+|\epsilon|)^{2}}\left(\frac{3 \beta}{2} \tau-\frac{9 \beta^{2} / 4-(\Delta+|\epsilon|)^{2}}{9 \beta^{2} / 4+(\Delta+|\epsilon|)^{2}}\right) \\
& \left.+\frac{2}{9 \beta^{2} / 4+(\Delta-|\epsilon|)^{2}}\left(\frac{3 \beta}{2} \tau-\frac{9 \beta^{2} / 4-(\Delta-|\epsilon|)^{2}}{9 \beta^{2} / 4+(\Delta-|\epsilon|)^{2}}\right)\right]+\mathcal{O}\left[\left(\frac{\beta}{|\epsilon|}\right)^{2}\right]
\end{aligned}
$$


Two remarks must be made about this expression. Firstly, the elastic component is of higher order than the other three peaks and does not appear here. This means the integrated intensity in the elastic component is much less than in the inelastic ones, and it becomes visible in the spectrum as $\tau \rightarrow \infty$ only because it is so narrow. Secondly, the $T_{1}$ dependence no longer appears explicitly, but is contained in the higher order terms. Indeed, one should compare (24) to the solutions (4.22) through (4.25) found by Carmichael and Walls (1976) for an atom in a strong field, observed for a finite amount of time after it has reached the steady state. Neglecting their exponentially decaying terms (since $\tau$ is assumed greater than a few lifetimes), one finds their results identical to (24). It is easy to see that there is a difference in the height of a peak at finite $\tau$ and in the stationary limit, and that this difference goes to zero like $1 / \tau$. Similarly, equation (24) makes it clear that the narrowing visible in the sequence of spectra in figure $3(b)$ is due to the increase in the length of the observation region. Therefore, even though the spectra in figure $3(b)$ are for atoms which have been in the interaction region for only a short time, the narrowing of the peaks should not be attributed to the recent turn-on of the interaction because it is independent of $T_{1}$.

To illustrate this point better, we have considered the spectra for a situation identical to that of figure $3(b)$, with the exception that the observation region was moved from $[0, \tau]$ to $[40,40+\tau]$. When they enter the observation region, the atoms have already been interacting with the field for so long ( 40 lifetimes) that no effects remain from the time when they entered the field. These spectra have not been reproduced here because they are virtually indistinguishable from the corresponding spectra for $T_{1}=0$ and the same $\tau$. Thus we conclude that the noticeable features in the sequence of spectra in figure $3(b)$ are not associated with the interaction time, but rather are a consequence of a finite observation region.

To see effects which are related to the turn-on of the interaction rather than caused by the limited observation interval, it is better to detune the applied field somewhat from resonance. In figure 4 we again show a sequence of spectra corresponding to the observation regions $[0, \tau]$. The atoms are assumed to be in the ground state when they enter the interaction region. The field parameters are $\epsilon=7 \beta$, $\alpha=-7 \beta$. For this field, the relative minima and maxima of the spectra form noticeable valleys and ridges. These follow curves which are rectangular hyperbolae in the $\Delta \tau$ plane, and they are especially pronounced on the right-hand side of the central peak. Although in the stationary limit the spectra are always symmetric about the incident field frequency, even when the detuning is non-zero, in the present case the lineshapes are noticeably asymmetric. These asymmetries consist of: (i) an enhanced sideband on the atomic resonance frequency side of the central peak; (ii) more pronounced oscillations between the central peak and the enhanced sideband than on the opposite side of the central peak; (iii) a slight displacement in the location of the central maximum from the applied field frequency toward the atomic frequency.

We must again consider whether these features are, in fact, due to the start of the atom-field interaction, and not to the finite observation region. This question can be most directly answered by fixing the length of the observation region and investigating the spectra for various values of $T_{1}$ (i.e. as the slit of fixed width is moved down the atomic beam). Such spectra are shown in figures $5(a)$ and $(b)$. The field parameters for both figures are the same as for figure 4 . In figure $5(a)$ the slit width corresponds to 4 lifetimes, and in figure $5(b)$ it is twice as wide, that is, 8 lifetimes. One can see that the larger value of $\tau$ (i) gives narrower peaks and 


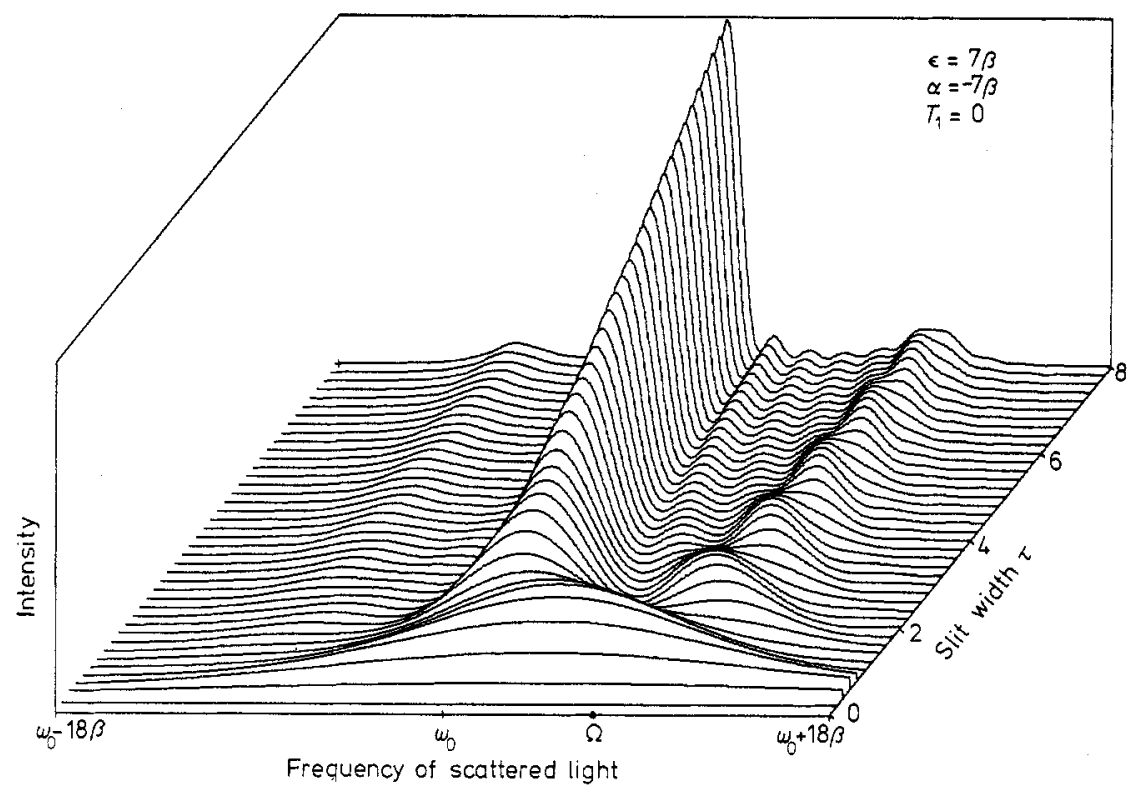

Figure 4. Emission spectra for various widths $\tau$ of a slit positioned at the front of the interaction region. The field is strong and tuned off-resonance. The spectra show asymmetric features due to the sudden turn-on of the interaction.

(ii) puts a larger number of oscillations in the spectrum. As the front edge of the observation region is moved back from the point where the atoms enter the laser beam, these two effects are unchanged. In contrast, the asymmetries discussed above, i.e. the enhanced sideband and oscillations on the atomic frequency side of the central peak, are easily seen to fade away as $T_{1}$ becomes larger. The shift of the central maximum also decreases as the slit is moved along the atomic beam away from the front of the interaction region. However, since in this particular case of figure $5(a)$ this shift is only about $\frac{1}{5} \beta$ even for $T_{1}=0$, this effect is not visible on these graphs. The longer the atoms have been in the interaction region, the less significant these asymmetries in the lineshape become. Thus they are properly referred to as turn-on features attributable to the sudden turn-on of the interaction.

It is important to note that these transient effects disappear much more slowly from the spectra in figure 4 than in figure 5. For $T_{1}$ about 4 lifetimes in figure 5 , the spectra have already approached very closely their asymptotic ( $T_{1}$ going to infinity) symmetric shape. The spectra in figure 4 , however, still show pronounced asymmetries at $\tau=4$ lifetimes. In fact, for $\tau=20$ the ratio of the sidebands is $1 \cdot 34: 1$, significantly greater than the one-to-one ratio found in the stationary limit. These two types of behaviour are easily understood by reference to expression (18) for $I_{\mathrm{D}}\left(\hat{k}, T_{1}, T_{1}+\tau, \omega_{0}+\Delta\right)$. Only those terms which are a function of $T_{1}$ can be responsible for the asymmetric spectral features which we have called turn-on effects. Obviously, those are just the terms in the double sum,

$$
\sum_{l=1}^{4} \sum_{m=2}^{4}\left[\frac{C_{l m} \exp \left(s_{m} T_{1}\right)}{\left(s_{l}-s_{m}+\mathrm{i} \Delta\right) \tau}\left(\frac{\exp \left[\left(s_{l}+\mathrm{i} \Delta\right) \tau\right]-1}{\left(s_{l}+\mathrm{i} \Delta\right)}-\frac{\exp \left(s_{m} \tau\right)-1}{s_{m}}\right)+\mathrm{CC}\right] .
$$



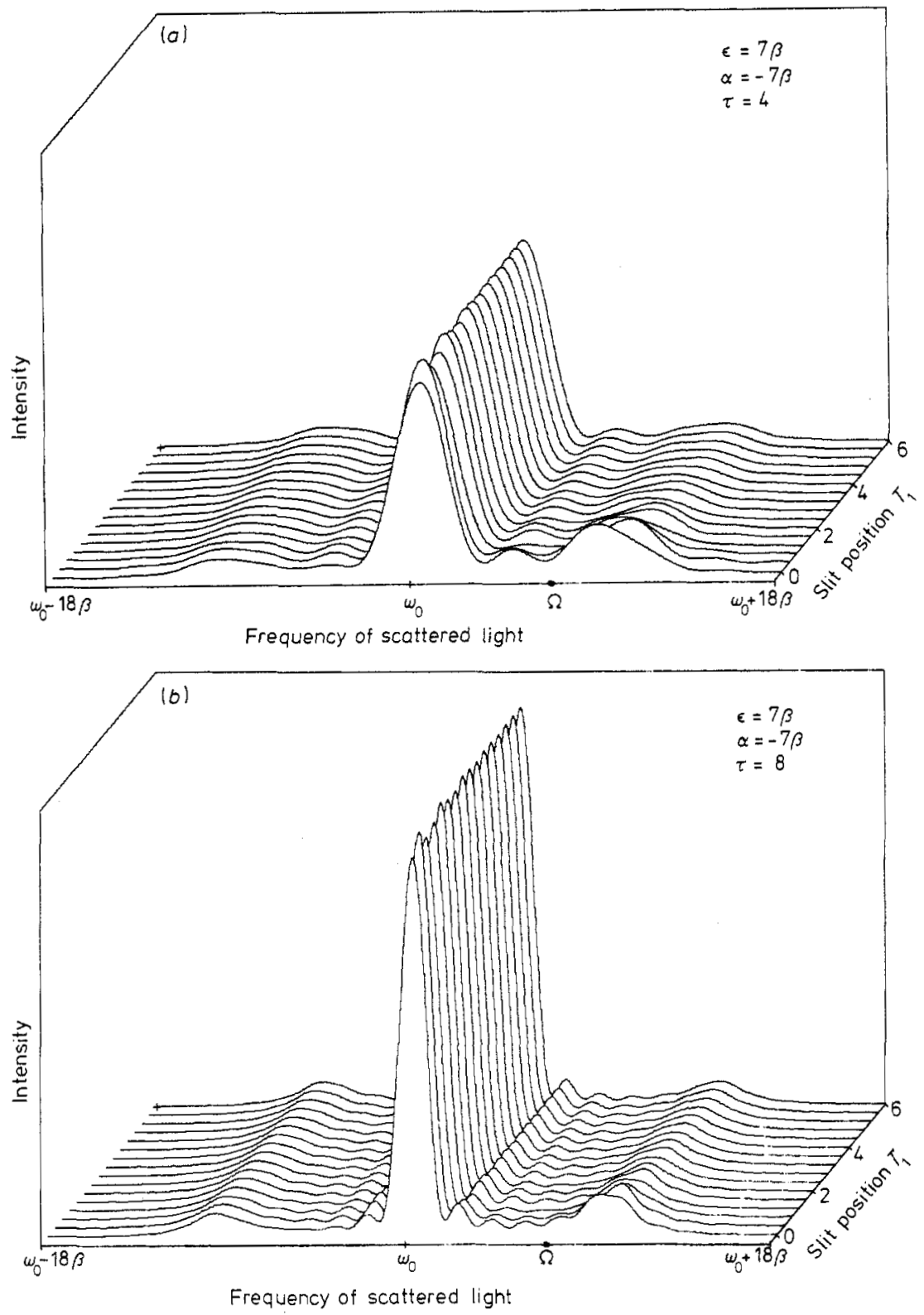

Figure 5. Emission spectra for a slit (a) 4 lifetimes wide and (b) 8 lifetimes wide as a function of position along the beam.

Recall that $\operatorname{Re}\left(s_{m}\right)<-\beta$ for $m=2,3,4$. Thus as $T_{1}$ increases in figures $5(a)$ and (b), the magnitude of the transient effects decreases exponentially. The behaviour is much different when $T_{1}$ is fixed and $\tau$ is increased, as in figure 4 . In this case the function in the large round brackets is purely oscillatory for $l=1$ (there is no damping in $\tau$ ), and so the contributions of these terms to the spectrum are inversely proportional to $\tau$. This is much slower than an exponential decrease, and, therefore, these transient effects can be significant even after many natural lifetimes. 
The specific turn-on effects which we have just described are strongly dependent upon the initial state of the atoms as they enter the laser field. Although we have considered each atom to be in its ground state, it was not necessary to do so. Equations (14) and (18) allow one to consider any possible initial density matrix for the atoms, and the particular choice can make a qualitative difference in the transient features of the spectrum. For example, for atoms initially in the excited state, the pronounced sideband on the atomic frequency side would be several times larger, resulting in an even more asymmetric lineshape. (It is reassuring to note that as $\epsilon \rightarrow 0$, this sideband becomes just the Lorentzian of a spontaneously decaying atom, located at the atomic frequency, as it should.) If one chooses for the initial state the proper coherent superposition of ground and excited states, for the same values of the parameters as in figures 4 and 5, one can find the opposite sideband to be initially more pronounced. Even when the applied field is exactly resonant, similar initial states can be found which lead to asymmetrical turn-on features. A consideration of the dressed states of a driven two-level atom (Stroud 1971) can provide one with an intuitive understanding of the origin of these transient effects. Because all these asymmetrical turn-on features basically are due to a non-equilibrium density matrix, their significance in the spectra as a function of $T_{1}$ and $\tau$ is the same as discussed above and illustrated in figures 4 and 5 . This is rigorously confirmed by reference to expression (25), since different initial conditions merely change the values of the coefficients $C_{l m}$ without affecting its functional dependence on $T_{1}$ and $\tau$.

\section{The Lorentz model approximation}

The asymmetries we have discussed are not necessarily quantum-mechanical features. For example, consider the two limits, first for very low field

$$
|\epsilon| \ll \beta
$$

and second for very large detuning

$$
|x| \gg|\epsilon| \text {. }
$$

In either limit the atom is not appreciably excited $\left(\left\langle\sigma_{22}(t)\right\rangle \ll 1\right)$ and one would, therefore, expect the Lorentz model of a classical damped harmonic oscillator driven by a classical field to be able to describe the atom. This identification with the Lorentz model has already been made in the stationary limit (see e.g. Heitler 1954), where the emission spectrum consists solely of the delta function at the incident field frequency. One can similarly derive a power spectrum for the Lorentz atom for short times from the proportionality relation

$$
I(\omega, \tau) \propto \frac{\left|\tilde{\mu}_{\tau}(\omega)\right|^{2}}{\tau}
$$

where $\tilde{\boldsymbol{\mu}}_{\tau}(\omega)$ is the truncated Fourier transform (in the sense of equations $(4 a)$ and $4(b))$ of the classical dipole moment $\mu(T)$ of the oscillator. The result is

$$
\begin{aligned}
I(\omega, \tau) \propto\left[\frac{2(1-\cos \Delta \tau)}{\Delta^{2} \tau}+\frac{1+\mathrm{e}^{-2 \beta \tau}-2 \mathrm{e}^{-\beta \tau} \cos [(\Delta+\alpha) \tau]}{\left[(\Delta+\alpha)^{2}+\beta^{2}\right] \tau}\right. \\
\\
\left.\quad+\left(\frac{\exp [-(\mathrm{i} \alpha+\beta) \tau]+1-\exp (\mathrm{i} \Delta \tau)-\exp \{-[\mathrm{i}(\Delta+\alpha)+\beta] \tau\}}{\mathrm{i} \Delta \tau[\mathrm{i}(\Delta+\alpha)+\beta]}+\mathrm{CC}\right)\right]
\end{aligned}
$$


where all the variables have already been defined previously (note that $T_{1}=0$ ). The initial conditions have been chosen to be

$$
\boldsymbol{\mu}(0)=\dot{\boldsymbol{\mu}}(0)=0 .
$$

When $\tau$ is much larger than a couple of lifetimes, the decaying terms in (29) can be approximated by zero; then

$$
\begin{aligned}
I(\omega, \tau) \underset{\tau \gg(1 / \beta)}{\longrightarrow} & \propto\left(\frac{2(1-\cos \Delta \tau)}{\Delta^{2} \tau}+\frac{1}{\tau\left[(\Delta+\alpha)^{2}+\beta^{2}\right]}\right. \\
& \left.-2 \frac{(1-\cos \Delta \tau)(\Delta+\alpha)+\beta \sin \Delta \tau}{\Delta \tau\left[(\Delta+\alpha)^{2}+\beta^{2}\right]}\right) .
\end{aligned}
$$

The first term is the elastic component which becomes a delta function for $\tau \rightarrow \infty$, and leads to the stationary spectrum. The second term is a Lorentzian at the atomic frequency which decreases as $1 / \tau$. The third term consists of the interferences between 'photons' emitted at the atomic and field frequencies; it also vanishes in $1 / \tau$ and cannot be large in the case when (27) is satisfied. Expressions identical to (29) and (31) can also be derived from equations (14) and (18) in the two limits and with the proper initial conditions mentioned above. Thus the identification with the Lorentz model has been extended to the non-stationary regime.

Equations (29) and (31) are instructive because they show how some of the transient features enter the spectrum in a simple explicit way. As an example, in figure 6 a sequence of emission spectra for the observation region $[0, \tau]$ and field parameters $\alpha=-20 \beta$ and $\epsilon=2 \beta$ are shown. Note the expected asymmetry with respect to the applied field frequency, particularly the pronounced sideband and oscillations on the side nearer the atomic frequency. For larger $\tau$ the elastic component approaches its delta-function limit and the other features die away in $1 / \tau$. All this is in agreement with relations (29) and (31).

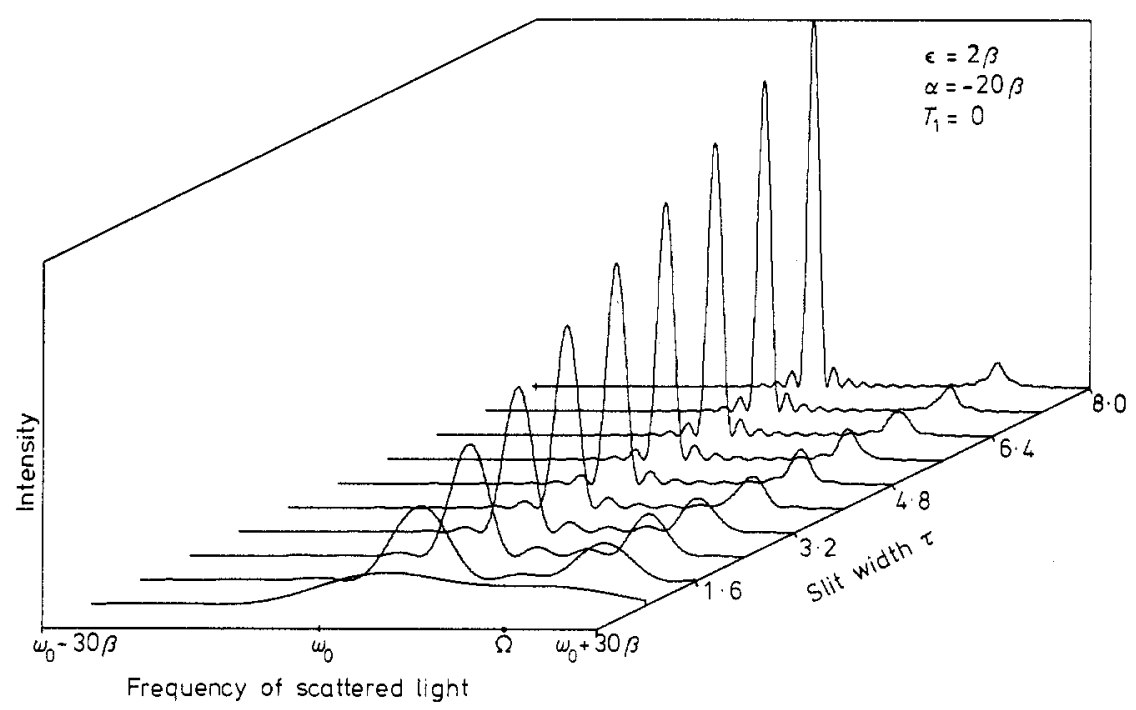

Figure 6. The Lorentz model limit as approached by tuning the field far from resonance. The observation is made on the front of the interaction region. 


\section{Remarks}

In this paper, we have shown that the quantity which is measured in a resonance fluorescence spectrum experiment is the total intensity, $I_{\mathrm{D}}\left(\hat{\boldsymbol{k}}, T_{1}, T_{1}+\tau, \omega_{0}+\Delta\right)$, as given by relation (18). It should be noted that this quantity is not proportional to the expression $\mathrm{d}\left(\left\langle n_{k \lambda}(t)\right\rangle\right) / \mathrm{d} t$ which is the rate of change of the number of photons in the mode $k\rangle$ due to the emission of an atom from time $\tau=0$ up to time $\tau$. This last expression has been considered in previous treatments (Mollow 1969, Agarwal 1974, Hassan and Bullough 1975) to yield the spectrum in the stationary limit $(\tau \rightarrow \infty)$. However, it would be erroneous to carry over this expression into the nonstationary regime. Indeed, this quantity becomes negative for some frequencies at small times, and has other properties quite different from those seen in experimentally measured spectra. This has already been pointed out by Herrmann et al (1973).

The emission spectrum can be properly related to the number of photons in the field mode $k \lambda$ at time $t_{2}$ due to the radiation of an atom from $t_{1}$ to $t_{2}$,

$$
\begin{aligned}
\left\langle n_{\boldsymbol{k} \lambda}\left(t_{2}, t_{1}\right)\right\rangle & =\left|g_{\boldsymbol{k} \lambda}\right|^{2} \int_{t_{1}}^{t_{2}} \mathrm{~d} t^{\prime \prime} \int_{t_{1}}^{t_{2}} \mathrm{~d} t^{\prime}\left\langle\sigma_{-}\left(t^{\prime}\right) \sigma_{-}\left(t^{\prime \prime}\right)\right\rangle \exp \left[-\mathrm{i} \omega_{k}\left(t^{\prime}-t^{\prime \prime}\right)\right] \\
& =\left|g_{k \lambda}\right|^{2} \int_{t_{1}}^{t_{2}} \mathrm{~d} t^{\prime \prime} \int_{t_{1}}^{t^{\prime \prime}} \mathrm{d} t^{\prime}\left\langle\sigma_{+}\left(t^{\prime}\right) \sigma_{-}\left(t^{\prime \prime}\right)\right\rangle \exp \left[-\mathrm{i} \omega_{k}\left(t^{\prime}-t^{\prime \prime}\right)\right]+\mathrm{CC}
\end{aligned}
$$

This is a generalization of the definition of $\left\langle n_{k i}(t)\right\rangle$ derived in RWS I. Comparing the above expression with equation (11), we see that it is related to the measured spectrum in the following way:

$$
I_{\mathrm{D}}\left(\hat{\boldsymbol{k}}, T_{1}, T_{1}+\tau, \omega_{0}+\Delta\right) \propto \frac{N A}{\tau} \sum_{\lambda}\left\langle n_{\boldsymbol{k} \hat{\lambda}}\left(T_{1}+\tau, T_{1}\right)\right\rangle .
$$

It is the number of photons emitted in the observation interval $\tau$ which is the quantity related to the experimentally measured spectrum, not $\mathrm{d}\left(\left\langle n_{k \lambda}(t)\right\rangle\right) / \mathrm{d} t$. If, however, one goes to the stationary limit, where the length $\tau$ of the observation region goes to infinity, one finds that the number of photons scattered into the mode $k \lambda$ grows linearly with $\tau$. In this limit,

$$
\frac{1}{\tau}\left\langle n_{\boldsymbol{k} \lambda}\left(T_{1}+\tau, T_{1}\right)\right\rangle=\frac{\mathrm{d}}{\mathrm{d} \tau}\left\langle n_{\boldsymbol{k} \lambda}(\tau)\right\rangle \quad \forall T_{1}, \tau \rightarrow \infty .
$$

For this reason, some previous calculations which defined the spectrum as the derivative of the photon number obtained correct results for the stationary limit.

From expression (32) it can be seen that $\left.\left\langle n_{\boldsymbol{k}}\right\rangle\left(t_{2}, t_{1}\right)\right\rangle$ is not a simple integral over the interval $\left[t_{1}, t_{2}\right]$ of an integrand which is independent of the end points. Thus the number of photons scattered in the interval $\left[t_{1}, t_{3}\right]$ is not just the sum of the photons scattered in the intervals $\left[t_{1}, t_{2}\right]$ and $\left[t_{2}, t_{3}\right]$. Rather, we find

$$
\begin{aligned}
\left\langle n_{\boldsymbol{k} \lambda}\left(t_{3}, t_{1}\right)\right\rangle= & \left\langle n_{\boldsymbol{k} \lambda}\left(t_{3}, t_{2}\right)\right\rangle+\left\langle n_{\boldsymbol{k} \lambda}\left(t_{2}, t_{1}\right)\right\rangle \\
& +\left(\left|g_{\boldsymbol{k} \lambda}\right|^{2} \int_{t_{1}}^{t_{2}} \mathrm{~d} t^{\prime} \int_{t_{2}}^{t_{3}} \mathrm{~d} t^{\prime \prime}\left\langle\sigma_{+}\left(t^{\prime}\right) \sigma_{-}\left(t^{\prime \prime}\right)\right\rangle \exp \left[-\mathrm{i} \omega_{k}\left(t^{\prime}-t^{\prime \prime}\right)\right]+\mathrm{cC}\right) .
\end{aligned}
$$

The additional term represents an interference between the photons emitted earlier with those emitted later, in the same way that light coming from two regions of 
a slit can interfere to give a different angular distribution of the light than would be found from summing the diffraction patterns due to each separate region. Although $\left\langle n_{\boldsymbol{k},}\left(t_{2}, t_{1}\right)\right\rangle$ is a positive definite quantity, its derivative is not.

It is interesting to note that though our calculations are fully quantum mechanical, some of the most surprising results can be explained in classical terms. For instance, the asymmetries in the spectrum related to the turn-on were shown to be derivable from the Lorentz model. Also, the ringing in the elastic component due to the finite observation region is just what one expects classically from the Fourier transform of a truncated sinusoid.

In conclusion, we have completely solved the two-level resonance fluorescence problem using the Heisenberg picture QED, and have derived the proper expression for the emission spectrum which would be measured in a realistic experiment. Our derivation has avoided any ad hoc assumptions concerning what quantity related to the quantized field is measured in such an experiment. This spectrum has been obtained not only as a function of the atomic and field parameters, but also as a function of two variables which are available to the experimenter: the slit position $T_{1}$ and observation interval $\tau$. Thus it correctly incorporates the possible effects of (i) a finite observation interval, (ii) observations made close to the point where the interaction was turned on, and (iii) arbitrary initial conditions for the atom at the start. All of the solutions have been obtained analytically, and a computer has been used only to generate graphical illustration of particular cases.

\section{References}

Agarwal G S 1974 Springer Tracts in Modern Physics vol 70 (Heidelberg: Springer) Born $\mathrm{M}$ and Wolf E 1970 Principles of Optics (New York: Pergamon)

Carmichael H J and Walls D F 1975 J. Phys. B: Atom. Molec. Phys. 8 L77-81

-1976 J. Phys. B: Atom. Molec. Phys. 9 1199-219

Cohen-Tannoudji C 1975 Laser Spectroscopy ed S Haroche et al (Berlin: Springer) pp 324-39

Gibbs H M and Venkatesan T N C 1976 Opt. Commun. 17 87-90

Hassan S S and Bullough R K 1975 J. Phys. B: Atom. Molec. Phys. 8 L147-52

Heitler W 1954 The Quantum Theory of Radiation (Oxford: Clarendon)

Herrmann J, Süsse K-E and Welsch D 1973 Ann. Phys., Lpz 30 37-54

Kimble H J and Mandel L 1976 Phys. Rev. A 13 2123-44

Mollow B R 1969 Phys. Rev: 188 1969-75

- 1975 Phys. Rev. A 12 1919-43

Renaud B, Whitley R M and Stroud C R Jr 1976 J. Phys. B: Atom. Molec. Phys. 9 L19-24

Rehler N E and Eberly J H 1971 Phys. Rev. A 3 1735-51

Schuda F and Stroud C R Jr 1973 Opt. Commun. $914-6$

Schuda F, Stroud C R Jr and Hercher M 1974 J. Phys. B: Atom. Molec. Phys. 7 L198-202

Smithers M E and Freedhoff H S 1974 J. Phys. B: Atom. Molec. Phys. 7 L432-5

Stroud C R Jr 1971 Phys. Rev. A 3 1044-52

Swain S 1975 J. Phys. B: Atom. Molec. Phys. 8 L437-41

Walther H 1975 Laser Spectroscopy ed S Haroche et al (Berlin: Springer) pp 358-69

Wu F Y, Grove R E and Ezekiel S 1975 Phys. Rev. Lett. 35 1426-9 